\title{
Growth competition between Halobacterium salinarium strain PHH1 and mutants affected in gas vesicle synthesis
}

\author{
Steven J. Beard, Paul K. Hayes and Anthony E. Walsby \\ Author for correspondence: Anthony E. Walsby. Tel: +44 1179287490 . Fax: +44 1179257374.
}

School of Biological Sciences, University of Bristol, Woodland Road, Bristol BS8 1UG, UK

\begin{abstract}
To investigate the role of the buoyancy provided by gas vesicles in the facultative anaerobe Halobacterium salinarium PHH1, the growth of a gasvacuolate $\left(\mathrm{Gv}^{+}\right)$strain in competition with two gas-vesicle-defective (Gvdef) mutants was examined. The $\mathrm{Gv}^{+}$strain synthesized gas vesicles throughout its growth cycle, and floated up to form a thick surface scum during the exponential growth phase in static culture. Mutant Gvdef1 produced significantly fewer gas vesicles than the $\mathrm{Gv}^{+}$strain in corresponding stages of growth, although in late stationary phase a small proportion of cells floated up to the surface of static cultures. Mutant Gvdefz had a much lower gas vesicle content in shaken culture and produced negligible amounts of gas vesicles in static culture. The $\mathrm{Gv}^{+}$and the two Gvdef strains grew equally well in shaken cultures, but in static cultures, where steep vertical gradients of oxygen concentration were established, Gvdef1 was outgrown by the $\mathbf{G v}^{+}$strain. $\mathbf{G v}^{\text {det2 }}$ outcompeted the $\mathbf{G v}^{+}$strain in shallow static cultures, perhaps because Gvdef2 carried a smaller protein burden, which offset the benefits of buoyancy. This selection for Gvdef2 was lost in deeper static cultures, although it could be restored by aerating static cultures from below. The results support the hypothesis that the role of buoyancy in halobacteria is to maintain cells at the more aerated surface of brine pools.
\end{abstract}

Keywords: buoyancy, gas vesicles, halobacteria, Halobacterium salinarium

\section{INTRODUCTION}

Diverse prokaryotes are made buoyant through the accumulation of gas vesicles (Walsby, 1994). The primary role of buoyancy in phototrophs is to maintain the micro-organisms either near the surface of the water or in the metalimnion of lakes, where they are exposed to favourable light intensities (Walsby, 1994). In other gas-vacuolate micro-organisms, such as the methanogen Methanosarcina barkeri (Archer \& King, 1984) and various sea-ice bacteria (Gosink \& Staley, 1995), the role of buoyancy is less obvious. Petter (1932) suggested that gas vesicles might be important to aerobic halobacteria in buoying them to the water surface, where the concentrations of oxygen are highest.

Petter's hypothesis may apply to other aerobic bacteria; it has been tested in laboratory experiments conducted

Abbreviations: $D_{c, 700}$ attenuance following gas vesicle collapse; $\Delta T$, decrease in turbidity following gas vesicle collapse; RGV, relative gas vesicle content. with the heterotrophic freshwater bacterium Prosthecomicrobium pneumaticum, an obligate aerobe that produces sufficient gas vesicles to render it positively buoyant at all stages of growth (Walsby, 1976). In static cultures, in which vertical gradients of oxygen concentration could develop, the wild-type strain outcompeted a gas-vesicle-deficient mutant. In shaken cultures (and in static growth vessels supplied with air at both the top and bottom surfaces) both strains grew at about the same rate. These competition experiments suggest that in $P$. pneumaticum the function of the gas vesicles is to buoy cells to the more aerated water surface (Walsby, 1976).

The extremely halophilic archaeon Halobacterium salinarium (formerly $H$. halobium) accumulates large numbers of gas vesicles and forms floating scums in static culture (Larsen et al., 1967). Rodriguez-Valera et al. (1985) measured oxygen concentrations as low as $0.50 \mathrm{mg} 1^{-1}$ in the solar salterns occupied by $H$. salinarium, whereas the concentration of oxygen in airsaturated freshwater is about $7 \mathrm{mg} \mathrm{l}^{-1}$ at the same 
temperature $\left(40^{\circ} \mathrm{C}\right)$. Such low oxygen concentrations are a consequence of the limited solubility of oxygen at high salt concentrations (Smith, 1928) and of respiration by micro-organisms present at high densities in these habitats (Rodriguez-Valera et al., 1985). H. salinarium is a facultative anaerobe: it can grow under microaerobic and anaerobic conditions by using alternative terminal electron acceptors (Oren, 1991), by fermenting arginine (Hartmann et al., 1980), or by using light-driven ion pumps, such as bacteriorhodopsin (Oesterhelt \& Stoeckenius, 1973; Rodriguez-Valera et al., 1983). In environments where there are steep gradients of oxygen concentration, however, gas vesicle production may provide an advantage to the halobacteria by allowing them to float to the brine surface, where aerobic respiration can occur, as Petter (1932) suggested.

This paper describes the first quantitative measurements of gas vesicle production and growth by Halobacterium salinarium strain PHH1. We also describe competition experiments using $H$. salinarium $\mathrm{PHH} 1$ (wild-type for gas vesicle synthesis; $\mathrm{Gv}^{+}$) and mutants affected in gas vesicle production, to investigate the role of the buoyancy provided by gas vesicles. $H$. salinarium strain $\mathrm{PHH} 1$ contains two gene clusters capable of directing gas vesicle synthesis, p-vac (located on an endogenous plasmid) and c-vac (located on the chromosome) (Englert et al., 1992). In the $\mathrm{Gv}^{+}$strain, expression of the p-vac cluster, which leads to the constitutive synthesis of spindle-shaped gas vesicles, represses expression of the c-vac cluster (Surek et al., 1988; Horne \& Pfeifer, 1989). The c-vac region is expressed only in gas-vesicledefective $\left(\mathrm{Gv}^{\mathrm{def}}\right)$ mutants, which carry rearrangements within the p-vac region, and this leads to the accumulation of cylindrical gas vesicles in the stationary phase of growth (Surek et al., 1988; Horne \& Pfeifer, 1989). A second class of gas vesicle mutants consists of those that produce no gas vesicles $\left(\mathrm{Gv}^{-}\right)$, presumably due to mutations within both p-vac and c-vac. Mutants affected in gas vesicle synthesis occur with a high frequency, reportedly up to $1 \%$ (Pfeifer $e t$ al., 1981), due mostly to insertions or deletions within p-vac (Pfeifer $e t$ al., 1988).

\section{METHODS}

Bacterial strains and growth conditions. Halobacterium salinarium strain PHH1 $\left(\mathrm{Gv}^{+}\right)$was provided by Dr Felicitas Pfeifer (Technische Hochschule Darmstadt, Germany). Cells were grown in darkness at $30^{\circ} \mathrm{C}$, in a basal salts medium $\left(4.0 \mathrm{M} \mathrm{NaCl}, 61 \mathrm{mM} \mathrm{MgSO}_{4}, 20 \mathrm{mM} \mathrm{KCl}\right.$ and $20 \mathrm{mM}$ Tris/ $\mathrm{HCl}, \mathrm{pH} 7.5)$ supplemented with peptone $(1.5 \%, \mathrm{w} / \mathrm{v}$; Oxoid) and solidified with $1.5 \%(\mathrm{w} / \mathrm{v})$ agar. Spread plates were sealed in plastic bags (to minimize desiccation) and incubated at $30^{\circ} \mathrm{C}$ for $2-3$ weeks. The plates were then stored at room temperature until it was possible to distinguish between colonies of the $\mathrm{Gv}^{+}$strain and gas-vesicle-deficient mutants: the $\mathrm{Gv}^{+}$strain produces creamy pink colonies, $\mathrm{Gv}^{-}$ mutants produce transparent colonies, and $\mathrm{Gv}^{\text {def }}$ mutants produce colonies that are initially transparent, but become opaque as the cells age (Simon, 1981). The biomass in liquid cultures was estimated from the attenuance at $700 \mathrm{~nm}$ (path length, $1 \mathrm{~cm}$ ) using an LKB Ultrospec spectrophotometer (model 4050, Biochrom). Samples were pressurized (to $0.80 \mathrm{MPa}$ ) to collapse all gas vesicles and then diluted with growth medium to give an attenuance that was within the linear region of the instrument.

Measurement of relative gas vesicle content. The relative gas vesicle content (RGV) of cell suspensions was estimated as the ratio $\Delta \mathrm{T} / D_{\mathrm{c}, 700}$, where $\Delta \mathrm{T}$ is the reduction in turbidity (measured in nephelometer units) observed on collapse of the gas vesicles, and $D_{c, 700}$ is the attenuance (at $700 \mathrm{~nm}$ ) of the cell suspension following gas vesicle collapse. Turbidity measurements were made with a pressure nephelometer (Walsby, 1973). Changes in RGV were determined for shaken cultures by taking samples at intervals; changes in RGV in static cultures were determined by setting up 33 cultures in $1.4 \mathrm{~cm}$ diameter glass tubes and, at each of 11 sampling times, taking samples from three replicate tubes that had been mixed (Figs 2 and 3). Inocula for these cultures were taken from starter cultures in the exponential growth phase.

Measurement of the oxygen concentration in static cultures. Measurements of oxygen concentration were made with a microelectrode which had a $700 \mu \mathrm{m}$ diameter tip (model 76822, Diamond General). The electrode was calibrated with Halobacterium growth medium $(23.3 \%, \mathrm{w} / \mathrm{v}, \mathrm{NaCl})$ containing sodium sulphite $(2 \%, w / v)$ to set the zero $\mathrm{O}_{2}$ concentration, and with medium which had been bubbled with air for $24 \mathrm{~h}$ to set the value corresponding to $100 \%$ saturation. Oxygen concentrations were measured as the tip of the electrode was moved downward in steps of $1 \mathrm{~mm}$ through a culture of the $\mathrm{Gv}^{+}$strain. The culture was grown in a glass tube $\left(2.3 \mathrm{~cm}\right.$ diameter) at $30^{\circ} \mathrm{C}$, but measurements were made at room temperature $\left(21^{\circ} \mathrm{C}\right)$. The concentration of oxygen in air-saturated $23.3 \%(\mathrm{w} / \mathrm{v}) \mathrm{NaCl}$ is calculated to be $67 \cdot 4 \mu \mathrm{mol} \mathrm{l}^{-1}$ using the data of Smith (1928).

Measurement of critical collapse pressure of gas vesicles. Critical collapse-pressure distributions for gas vesicles were determined by pressure nephelometry (Walsby, 1973) using cells suspended in the basal salts medium. Mean critical pressures were calculated as described by Walsby \& Bleything (1988).

Electron microscopy of cell lysates. Small quantities of cell material were scraped from agar plates and applied to the edge of pioloform-coated copper grids. The grids were floated face down on drops of water and then drained with filter paper and air-dried. Specimens were examined in a JEOL transmission electron microscope.

Growth competition in batch culture. $H$. salinarium $\mathrm{PHH}$ $\left(\mathrm{Gv}^{+}\right)$was grown in competition with each of the gas-vesicledefective mutants. All incubations were performed in darkness to avoid the complication of phototrophic growth. Cells were initially grown in $100 \mathrm{ml}$ batch cultures in $250 \mathrm{ml}$ Erlenmeyer flasks either shaken at 100 r.p.m. or left static. Subsequently, cells were cultured in $2.4 \mathrm{~cm}$ diameter glass tubes in which the lower end was either closed, or sealed by dialysis membrane, which was clamped in position by a glass chamber containing gas inlet and outlet tubes (Walsby, 1976). The gas inlet tube was connected to a supply of sterile moistened air. Both types of culture vessel were clamped in an upright position and contained $9 \mathrm{~cm}$ layers of liquid medium. Inocula for the competition experiments were taken from separate starter cultures in the exponential growth phase to give cell suspensions containing approximately equal numbers of $\mathrm{Gv}^{+}$and mutant cells; cell concentrations were determined using a Weber counting chamber. At various stages in the growth cycle, samples $(0.05 \mathrm{ml})$ were removed from the cultures, serially diluted in growth medium, and plated for subsequent 
counting. The static cultures were mixed before samples were removed. The numbers of $\mathrm{Gv}^{+}, \mathrm{Gv}^{\text {def }}$ and $\mathrm{Gv}^{-}$colonies were counted after they had grown sufficiently to allow the differences in their turbidity to be distinguished.

\section{RESULTS}

\section{Isolation of mutants affected in gas vesicle synthesis}

On agar plates, gas vesicle-defective $\left(G v^{\text {def }}\right)$ mutants of $H$. salinarium $\mathrm{PHH} 1$ appeared as reddish-pink colonies amongst the creamy-pink colonies of the $\mathrm{Gv}^{+}$strain. Inspection under the phase-contrast microscope showed that cells from a purified reddish-pink colony contained smaller amounts of gas vesicles, which were identified as pressure-sensitive, light-refractile bodies. This mutant was designated $\mathrm{Gv}^{\mathrm{def} 1}$. Examination in the electron microscope of lysed stationary-phase cells revealed that the $\mathrm{Gv}^{+}$strain contained predominantly (>99\%) spindle-shaped gas vesicles, whereas the $\mathrm{Gv}^{\text {def1 }}$ mutant contained mainly (about $55 \%$ ) cylindrical gas vesicles. In static liquid cultures, $\mathrm{Gv}^{+}$cells floated up to the surface during the exponential and stationary growth phases to form a thick film. Under identical growth conditions cells of $\mathrm{Gv}^{\text {def1 }}$ were suspended throughout the cultures, although some cells floated up to form a surface scum in late stationary phase. A second gasvesicle-defective mutant $\left(\mathrm{Gv}^{\text {dei2 }}\right)$ was isolated from a culture of $G v^{\text {def1 }}$. Initially $G v^{\text {def } 2}$ appeared to exhibit a $\mathrm{Gv}^{-}$phenotype since it produced orange transparent colonies in which no gas vesicles were observed. However, the pressure-sensitive turbidity detected in shaken cultures of $\mathrm{Gv}^{\mathrm{def} 2}$ indicated the presence of small amounts of gas vesicles. In static cultures, cells of $\mathrm{Gv}^{\text {def2 }}$ sank to the bottom of the growth vessel and remained there throughout the growth cycle, although in some old cultures a diffuse scum appeared in late stationary phase. Cells that were removed from these scums lacked gas vesicles and may have accumulated at the surface by swimming, since $H$. salinarium possesses flagella and has been shown to be positively aerotactic (Stoeckenius et al., 1988).

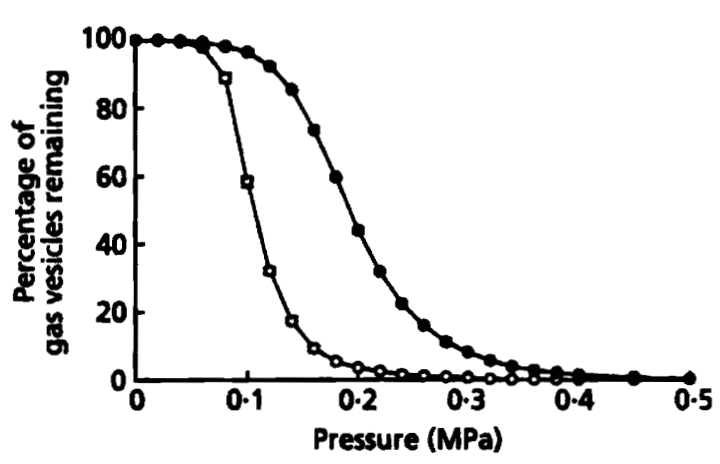

Fig. 1. Critical pressure distributions for gas vesicles in the $\mathrm{Gv}^{+}$ (O) and $\mathrm{Gv}^{\text {def1 }}(\mathrm{O})$ strains of H. salinarium PHH1. Means and standard deviations of five $\left(\mathrm{Gv}^{+}\right)$or three (Gvdef1) measurements are plotted (most of the error bars are smaller than the symbols).
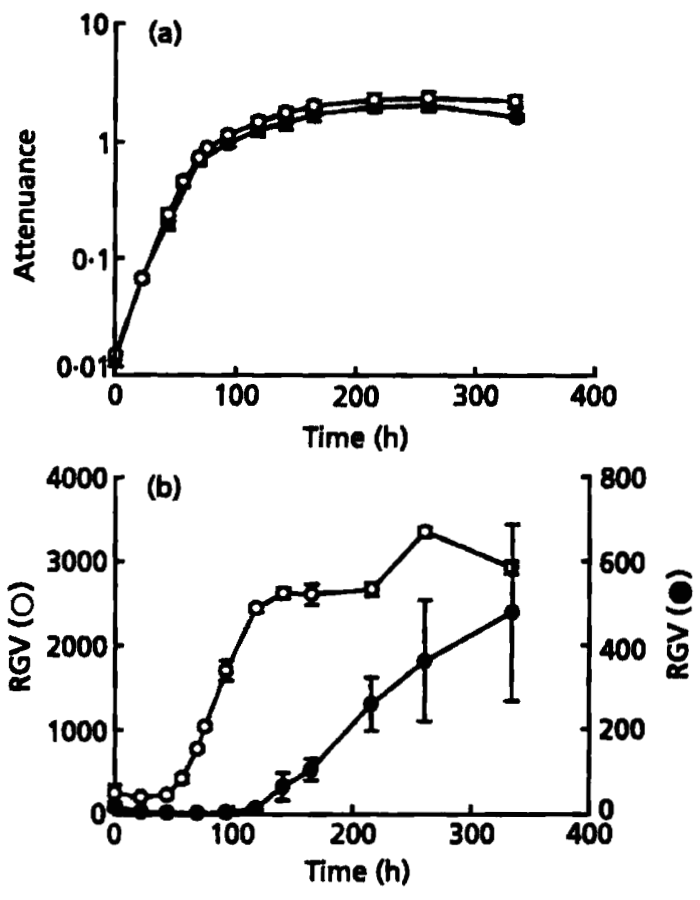

Fig. 2. Changes in (a) biomass and (b) RGV of the $\mathrm{Gv}^{+}$strain (O) and gas-vesicle-defective mutant Gvdef1 (O) when grown in shaken culture. Means and standard deviations of three measurements are plotted. Note the differences in the scales in (b).

\section{Gas vesicle critical pressures}

The critical pressure distributions of gas vesicles in cells from stationary-phase cultures of the $\mathrm{Gv}^{+}$and $\mathrm{Gv}^{\text {def1 }}$ strains were determined (Fig. 1). As in other organisms (Walsby, 1971; Walsby \& Bleything, 1988), the critical pressures of the gas vesicles in $H$. salinarium exhibited a broad normal distribution about a well-defined mean. The mean critical pressure of gas vesicles in the $\mathrm{Gv}^{+}$ strain was $0 \cdot 1138 \pm 0.0013 \mathrm{MPa}(95 \%$ confidence level, $n=5$ ), with a standard deviation of the distribution of $0.0396 \mathrm{MPa}$. The mean critical pressure of gas vesicles in $\mathrm{Gv}^{\text {def1 }}$ was $0.2012 \pm 0.0004 \mathrm{MPa}(95 \%$ confidence level, $n=3$ ), with a standard deviation of the distribution of 0.0679 $\mathrm{MPa}$.

\section{Growth and gas vesicle content of the $\mathrm{Gv}^{+}$and $\mathrm{Gv}^{\text {def }}$ strains in shaken and static cultures}

Measurements were made of the growth and gas vesicle content of the $\mathrm{Gv}^{+}$and $\mathrm{Gv}^{\text {def1 }}$ strains, grown in $2.6 \mathrm{~cm}$ deep cultures that were either shaken or left static. The growth curves were broadly similar for the two strains studied (Figs $2 a$ and $3 a$ ), with minimum mean doubling times of about $10 \mathrm{~h}$ in both shaken and static culture. Although the initial growth rates were similar in shaken and static culture, these rates were not sustained for so long in static culture, perhaps because the cultures became oxygen-limited. 

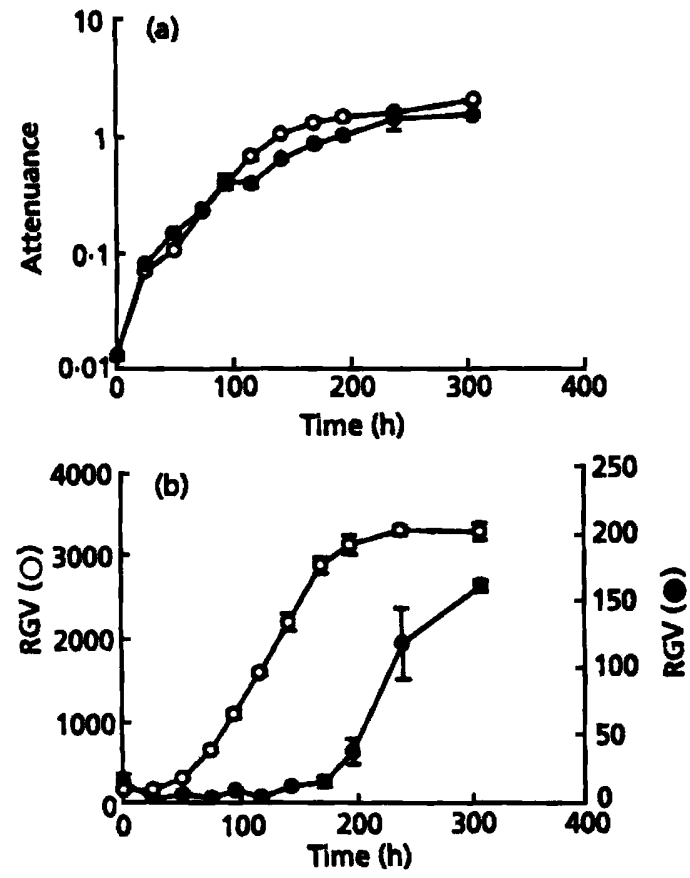

Fig. 3. Changes in (a) biomass and (b) RGV of the Gv+ strain (O) and gas-vesicle-defective mutant Gvdef1 $(O)$ when grown in static culture. Other details as in Fig. 2.

Changes in the relative gas vesicle content (RGV) demonstrated that gas vesicles were synthesized throughout the growth cycle of the $\mathrm{Gv}^{+}$strain in both shaken and static culture (Figs $2 \mathrm{~b}$ and $3 \mathrm{~b}$ ). In shaken culture (Fig. 2b) the RGV of the $\mathrm{Gv}^{+}$strain did not change significantly during the first $44 \mathrm{~h}$ of the exponential phase $(P=0.673)$, which indicates that the rate of gas vesicle production matched the exponential growth rate. Thereafter the RGV increased as the growth rate decreased. A broadly similar pattern of gas vesicle production was observed when the $\mathrm{Gv}^{+}$strain was grown in static culture (Fig. 3b). The RGV increased at a higher rate in shaken culture (compare the slopes in Figs $2 \mathrm{~b}$ and $3 \mathrm{~b}$ ). However, if the same measurements of RGV are plotted against culture biomass (Fig. 4a) rather than versus age of the culture, it is seen that the rise in gas vesicle formation occurs at an earlier stage of biomass development in static culture.

The amounts of gas vesicles accumulated in $G v^{\text {def1 }}$ were much less than in the $\mathrm{Gv}^{+}$strain (Figs $2 \mathrm{~b}$ and $3 \mathrm{~b}$ ). The highest mean RGV measured for $\mathrm{Gv}^{\mathrm{def1}}\left(\Delta \mathrm{T} / D_{\mathrm{c}, \mathbf{7 0 0}}=\right.$ 478) was approximately sevenfold less than the highest value measured for the $\mathrm{Gv}^{+}$strain $\left(\Delta \mathrm{T} / D_{\mathrm{c}, 700}=3347\right)$. In both shaken and static cultures (Figs $2 \mathrm{~b}$ and $3 \mathrm{~b}$ ) the RGV of $G v^{\text {def1 }}$ remained low $(\leqslant 25)$ throughout the exponential growth phase. The values of RGV were, however, higher than would have been expected if no gas vesicle synthesis occurred during exponential growth and the gas vesicles present in the cells of the inoculum were simply diluted out between siblings. The RGV
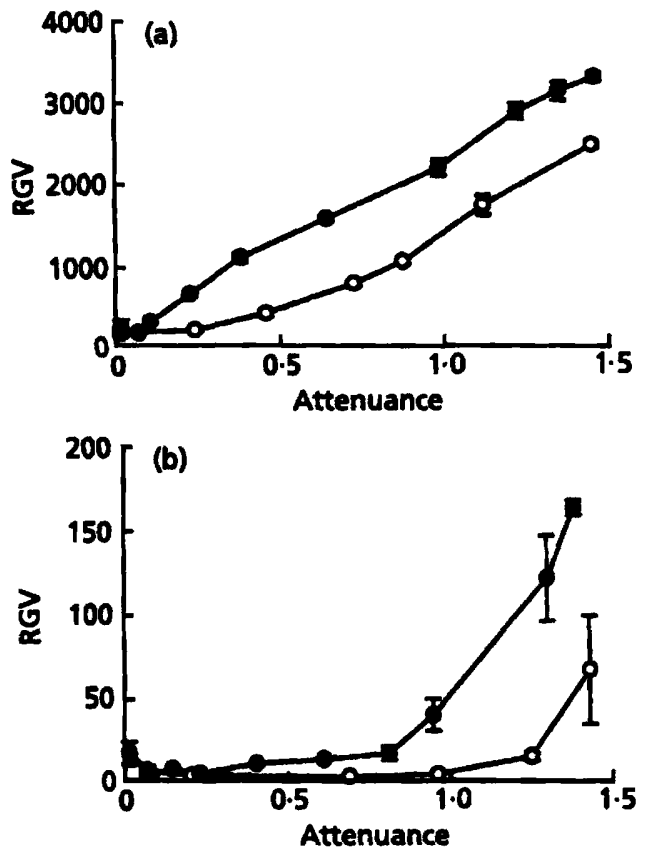

Fig. 4. Changes in the RGV as a function of the culture biomass of shaken (O) and static (O) cultures of the $\mathrm{Gv}^{+}$strain (a) and the gas-vesicle-defective mutant $\mathrm{Gv}^{\text {defi }}$ (b). These measurements are the same as those presented in Figs 2 and 3. Means and standard deviations of three measurements are plotted.

increased considerably during the stationary phase in both shaken and static culture (Figs $2 b$ and $3 b$ ). As observed for the $\mathrm{Gv}^{+}$strain, the $\mathrm{RGV}$ of $\mathrm{Gv}^{\text {defl }}$ began to increase at an earlier stage of biomass development in static culture than it did in shaken culture (Fig. $4 b$ ).

Growth curves obtained for $\mathrm{Gv}^{\text {def2 }}$ (data not shown) were similar to those obtained for the $\mathrm{Gv}^{+}$and $\mathrm{Gv}^{\text {defi }}$ strains, with minimum mean doubling times of about $10 \mathrm{~h}$ in shaken culture, and $9 \mathrm{~h}$ in static culture. Gas vesicles were detected in the late stationary phase of the shaken cultures at $0.5 \%$ of the level in the $\mathrm{Gv}^{+}$strain, and in these cultures the RGV varied considerably $\left\langle\Delta \mathrm{T} / D_{\mathrm{c}, 700}=18 \cdot 3 \pm 25 \cdot 1, n=3\right)$.

\section{Investigation of the vertical gradient of oxygen concentration in a static culture of the $\mathbf{G v}^{+}$strain}

A steep vertical gradient of oxygen concentration was measured in a static culture of the $\mathrm{Gv}^{+}$strain (Fig. 5). The culture, which was in the exponential phase of growth $\left(D_{c, 700}=0.375\right)$, had an RGV of $1.6 \times 10^{2}$ and contained a thin layer of buoyant cells at the surface. The concentration of oxygen at the surface of the culture was $66 \%$ of that in air-saturated growth medium, which corresponds to $45 \mu \mathrm{mol} 1^{-1}$. At a depth of $1 \mathrm{~mm}$, however, the oxygen concentration was only $7.3 \%$ saturation (approximately $5 \mu \mathrm{mol} 1^{-1}$ ). The oxygen concentration then fell gradually over the next $34 \mathrm{~mm}$ to reach $3.1 \%$ saturation (approximately $2 \mu \mathrm{mol}^{-1}$ ). 


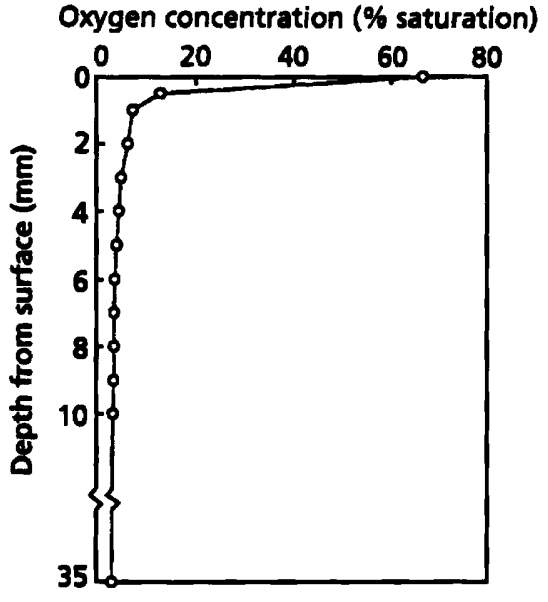

Fig. 5. Vertical gradient of oxygen concentration (percentage saturation) in a static culture of the $\mathrm{Gv}^{+}$strain. The concentration of oxygen corresponding to $100 \%$ saturation in a $23.3 \%(w / v) ~ N a C l$ solution is $67.4 \mu \mathrm{mol} \mathrm{I}^{-1}$ (see Methods).

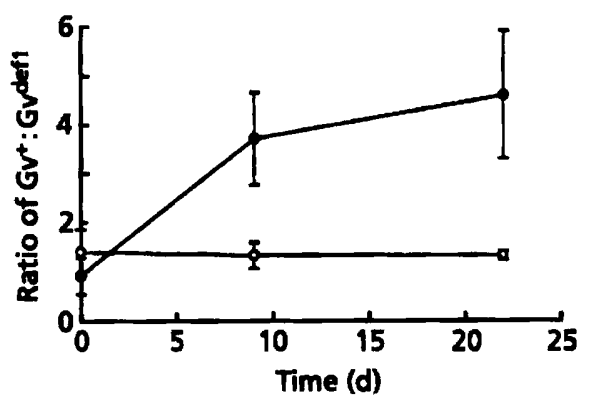

Fig. 6. Ratio of $\mathrm{Gv}^{+}: \mathrm{Gv}^{\text {def1 }}$ strains in shaken (O) and static (O) cultures after different times. Means and standard deviations of three measurements are plotted.

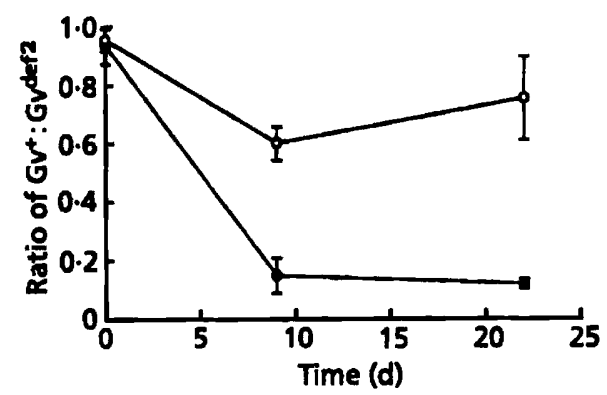

Fig. 7. Ratio of $\mathrm{Gv}^{+}: \mathrm{Gv}^{\operatorname{det} 2}$ strains in shaken $(\mathrm{O})$ and static cultures (O) after different times. Means and standard deviations of three measurements are plotted.

\section{Growth competition between the $\mathbf{G v}^{+}$and $\mathbf{G v}^{\text {def }}$ strains in shaken and static cultures}

$\mathrm{The}_{\mathrm{Gv}}{ }^{+}$strain was grown in competition with either $G v^{\text {def } 1}$ or $G v^{\text {def } 2}$ in $2.6 \mathrm{~cm}$ deep shaken and static cultures. When the $\mathrm{Gv}^{+}$and $\mathrm{Gv}^{\text {det1 }}$ strains were grown

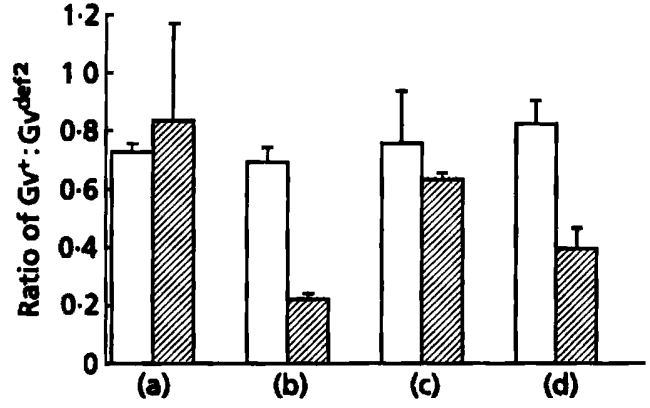

Fig. 8. Ratio of $\mathrm{Gv}^{+}: \mathrm{Gv}^{\text {def2 }}$ strains when grown together in shaken (a) and static (b) flasks, and in special culture vessels supplied with air at the top (c), or at top and bottom (d). Open bars, ratio in the inoculum; hatched bars, ratio after $10 \mathrm{~d}$ incubation. Means and standard deviations of three measurements are given.

together in shaken culture (Fig. 6) the proportions of the two strains did not change significantly over a $22 \mathrm{~d}$ period $(P=0.962)$. In static culture, however, $\mathrm{Gv}^{\text {def1 }}$ was outgrown by the $\mathrm{Gv}^{+}$strain (Fig. 6).

Two sets of experiments were performed to investigate the competition between the $\mathrm{Gv}^{+}$and $\mathrm{Gv}^{\text {def2 } 2}$ strains. In the first experiment (Fig. 7) we detected a small, but significant decrease in the ratio of $\mathrm{Gv}^{+}: \mathrm{Gv}^{\text {def2 }}$ after $9 \mathrm{~d}$ in shaken culture $(P=0.003)$, although after $22 \mathrm{~d}$ the ratio was not significantly different from that in the inoculum $(P=0 \cdot 150)$. There was no significant change in the ratio over the course of the second experiment in shaken culture $(P=0.630)$ (Fig. 8a). In static flasks, however, the $\mathrm{Gv}^{+}$strain was outgrown by Gv ${ }^{\mathrm{def} 2}$ (Figs 7 and $8 \mathrm{~b}$ ). This result suggested that the burden of producing gas vesicles in the $\mathrm{Gv}^{+}$strain resulted in a lower growth rate. In the second set of experiments additional cultures were grown in static vessels that contained $9 \mathrm{~cm}$ deep layers of growth medium, and in these cultures there was no significant change in the ratio of $\mathrm{Gv}^{+}: \mathrm{Gv}^{\mathrm{def2}}(P=0.360)$ (Fig. 8c). This suggested that growth of $\mathrm{Gv}^{\text {dep2 }}$ was limited by the low oxygen tension at the bottom of the deeper cultures and that this counteracted the intrinsically high growth rate shown in Fig. 8(b). Further $9 \mathrm{~cm}$ deep cultures were grown in special culture vessels in which air was supplied to both the top and bottom. In these cultures the growth advantage of $\mathrm{Gv}^{\text {def2 }}$ was partially restored (Fig. 8d), which indicated that growth of $\mathrm{Gv}^{\text {def2 }}$ was indeed limited by the reduced availability of oxygen in the deeper cultures (Fig. 8c). The growth medium used in this study could not support the growth of $H$. salinarium under anaerobic conditions: cultures that were sparged with oxygen-free nitrogen failed to grow.

\section{Investigation of the contributions of mutation and conjugation to the results of the competition experiments}

The ratio of $\mathrm{Gv}^{+}: \mathrm{Gv}^{\text {def }}$ strains could be affected by three processes: selection, mutation and the conjugal transfer of DNA encoding the gas-vacuolate phenotype. To 
investigate the possibility that changes in the mutation frequency may have influenced our results we measured the frequencies with which gas vesicle mutants appeared in clonal cultures of each of the three strains. After growing the cultures for $22 \mathrm{~d}$ in shaken and static flasks, cells were plated and the numbers of $\mathrm{Gv}^{+}, \mathrm{Gv}^{\text {def }}$ and $\mathrm{Gv}^{-}$ colonies were counted. For each strain, colonies exhibiting an altered gas vesicle phenotype appeared at a frequency of less than $1 \times 10^{-2}$. From this it was concluded that mutation made a negligible contribution to the changes in the ratio of $\mathrm{Gv}^{+}: \mathrm{Gv}^{\mathrm{def}}$ that were observed in the competition cultures. To determine whether the p-vac gene cluster could be transferred from the $\mathrm{Gv}^{+}$strain to $\mathrm{Gv}^{\text {def1 }}$ by conjugation we grew the $\mathrm{Gv}^{+}$ strain in competition with an anisomycin-resistant derivative of $\mathrm{Gv}^{\text {defl }}$, and plated cells on medium containing anisomycin $\left(8 \mu \mathrm{g} \mathrm{ml}^{-1}\right)$. Only colonies with a $\mathrm{Gv}^{\mathrm{de}}$ phenotype grew on this medium, which indicated that there was no detectable transfer of $\mathrm{p}$ - $v a c$ from the $\mathrm{Gv}^{+}$strain to $\mathrm{Gv}^{\text {def1 }}$.

\section{DISCUSSION}

The gas-vesicle-defective mutant $\mathrm{Gv}^{\text {def1 }}$ contained predominantly cylindrical gas vesicles, which were stronger than the spindle-shaped gas vesicles produced by the $\mathrm{Gv}^{+}$strain (Fig.1). These observations are consistent with those of Hayes \& Walsby (1986), who demonstrated an inverse relationship between the width and strength of gas vesicles in cyanobacteria. Walsby \& Bleything (1988) demonstrated that wider gas vesicles have a higher ratio of gas space to wall volume and are therefore more efficient in providing buoyancy. This is the basis of the hypothesis that there has been natural selection of gas vesicles with the maximum width allowed by the requirement to withstand pressure (Hayes \& Walsby, 1986). Halobacterial gas vesicles are the widest (Larsen et al., 1967; Stoeckenius \& Kunau, 1968; Simon, 1981) and weakest (this study; Walsby, 1971) known. They are strong enough to withstand the combination of the low hydrostatic pressures and negligible cell turgor pressure in halobacteria growing in shallow brine pools (Walsby, 1994). The stronger, cylindrical gas vesicles of gas-vesicle-defective mutants may be an adaptation to provide buoyancy in deeper brine lakes, but there has been no investigation of this.

The patterns of gas vesicle production seen in the $\mathrm{Gv}^{+}$ and $\mathrm{Gv}^{\mathrm{def}}$ strains of $H$. salinarium (Figs 2 and 3 ) correlate well with the patterns of p-gvpA and c-gvpA gene expression $(g \nu p A$ encodes the major gas vesicle protein, GvpA): in the $\mathrm{Gv}^{+}$strain maximal levels of $\mathrm{p}$ g $v p A$ mRNA were detected during the exponential growth phase, and in p-vac deletion mutants maximal levels of c-gvpA mRNA were detected in stationary phase (Horne \& Pfeifer, 1989). The observations that the RGV of both the $\mathrm{Gv}^{+}$and $\mathrm{Gv}^{\text {def } 1}$ strains began to increase at an earlier stage in the growth cycle when grown in static culture (as compared to when grown in shaken culture) indicate that gas vesicle synthesis may have been induced in response to the lower concen- trations of oxygen dissolved in the static cultures. This has also been suggested by Horne $\&$ Pfeifer (1989), who have stated that in Halobacterium sp. SB3 there may be greater gas vesicle production under anaerobic growth conditions. Furthermore, Yang \& DasSarma (1990) demonstrated that aeration significantly reduces the transcriptional induction of gvpA in $H$. salinarium NRC-1. Gas vesicle synthesis in Haloferax mediterranei was shown to be stimulated by growth at higher salt concentrations, in which the dissolved oxygen concentration would have been lower (Englert et al., 1990). These observations suggest that gas vesicle production in halobacteria is stimulated in oxygen-limited environments.

Steep gradients of oxygen concentration were shown to exist in the $2.6 \mathrm{~cm}$ deep static cultures of the $\mathrm{Gv}^{+}$strain (Fig. 5). The selection in static culture for the $\mathrm{Gv}^{+}$strain over the gas-vesicle-defective mutant $\mathrm{Gv}^{\text {def1 }}$ (Fig. 6) supports the hypothesis of Petter (1932) that in unstirred habitats it is advantageous for halobacteria to float to the more-aerated brine surface. Similar results were obtained in competition experiments conducted with the gas-vacuolate wild-type and gas-vesicle-deficient mutants of Prosthecomicrobium pneumaticum (Walsby, 1976). It is likely that the buoyancy provided by gas vesicles benefits the growth of $P$. pneumaticum throughout its growth cycle, since the cells are positively buoyant at all stages of growth (Walsby, 1976). H. salinarium, in contrast, achieves buoyancy only in the late exponential and stationary growth phases, which suggests that gas vesicles may be more important in the survival or dispersal of this organism. In the natural environment, gas vesicles must confer a selective advantage upon $H$. salinarium since the p-vac gene cluster has not been lost despite the extremely high frequency of p-vac mutants (Pfeifer et al., 1988).

An explanation is needed for the different results obtained in the competition experiments with the two $\mathrm{Gv}^{\mathrm{def}}$ strains. The Gv ${ }^{\text {def2 }}$ mutant produced negligible amounts of gas vesicles in static culture and therefore would have carried a smaller protein burden than either the $\mathrm{Gv}^{+}$or $\mathrm{Gv}^{\text {defi }}$ strains. This appeared to offset any advantage that the $\mathrm{Gv}^{+}$strain may have had in floating nearer the surface of the culture (Fig. 8b). This explanation is supported by the finding that net selection of the $\mathrm{Gv}^{\text {def2 }}$ mutant did not occur in deeper cultures where a greater proportion of the non-buoyant mutant cells were further from the oxygenated surface (Fig. 8c). The reason why $\mathrm{Gv}^{\text {def } 2}$ was not selected for in shaken culture (Fig. 8a) may have been that the $\mathrm{Gv}^{+}$strain produced fewer gas vesicles in the early stages of growth in shaken culture (Fig. 4a). In deeper cultures the advantage enjoyed by the $G v^{\text {def2 }}$ mutant was partially restored by providing aerobic conditions at the bottom surface (Fig. 8d). Therefore, it seems likely that gas vesicles become progressively more beneficial to the growth of $H$. salinarium as the depth of the culture increases (and the availability of oxygen decreases). There have been no comparable studies on how the growth rates of other gas-vacuolate micro-organisms 
are affected by the proportions of protein that are diverted into gas vesicle production. However, it has proved to be difficult to demonstrate the effects of a protein burden on the growth rate of Escherichia coli (Koch, 1983).

\section{ACKNOWLEDGEMENTS}

We would like to thank Mrs Avril Avery for technical assistance and Dr Felicitas Pfeifer for helpful discussions and the kind gift of $H$. salinarium strain PHH1. We are grateful to Professor John Parkes and $\mathrm{Mr}$ Alistair Lawson of the Department of Geology, University of Bristol, for assisting us with the measurements of oxygen concentration. This work was supported by a grant GR3/8987 from the NERC.

\section{REFERENCES}

Archer, D. B. \& King, N. R. (1984). Isolation of gas vesicles from Methanosarcina barkeri. J Gen Microbiol 130, 167-172.

Englert, C., Horne, M. \& Pfeifer, F. (1990). Expression of the major gas vesicle protein gene in the halophilic archaebacterium Haloferax mediterranei is modulated by salt. Mol Gen Genet 222, 225-232.

Englert, C., Kruger, K., Offner, S. \& Pfeifer, F. (1992). Three different but related gene clusters encoding gas vesicles in halophilic archaea. J Mol Biol 227, 586-592.

Gosink, J. J. \& Staley, J. T. (1995). Biodiversity of gas vacuolate bacteria from Antarctic sea ice and water. Appl Environ Microbiol 61, 3486-3489.

Hartmann, R., Sickinger, H.-D. \& Oesterhelt, D. (1980). Anaerobic growth of halobacteria. Proc Natl Acad Sci USA 77, 3821-3825.

Hayes, P. K. \& Walsby, A. E. (1986). The inverse correlation between width and strength of gas vesicles in cyanobacteria. $\mathrm{Br}$ Phycol J 21, 191-197.

Horne, M. \& Pfeifer, F. (1989). Expression of two gas vacuole protein genes in Halobacterium halobium and other related species. Mol Gen Genet 218, 437-444.

Koch, A. L. (1983). The protein burden of lac operon products. $J$ Mol Evol 19, 455-462.

Larsen, H., Omang, S. \& Steensland, H. (1967). On the gas vacuoles of the Halobacteria. Arch Mikrobiol 59, 197-203.

Oesterhelt, D. \& Stoeckenius, W. (1973). Functions of a new photoreceptor membrane. Proc Natl Acad Sci USA 70, 2853-2857.

Oren, A. (1991). Anaerobic growth of halophilic archaeobacteria by reduction of fumarate. J Gen Microbiol 137, 1387-1390.

Petter, H. F. M. (1932). Over roode en andere bakteriën gezouten visch. Doctoral thesis, University of Utrecht, Utrecht, The Netherlands.

Pfeifer, F., Weidinger, G. \& Goebel, W. (1981). Genetic variability in Halobacterium halobium. J Bacteriol 145, 375-381.
Pfeifer, F., Blaseio, U. \& Ghahraman, P. (1988). Dynamic plasmid populations in Halobacterium halobium. J Bacteriol $\mathbf{1 7 0}$, 3718-3724.

Rodriguez-Valera, F., Nieto, J. J. \& Ruiz-Berraquero, F. (1983). Light as an energy source in continuous cultures of bacteriorhodopsin-containing halobacteria. Appl Environ Microbiol 45, 868-871.

Rodriguez-Valera, F., Ventosa, A., Juez, G. \& Imhoff, J. F. (1985). Variation of environmental features and microbial populations with salt concentrations in a multi-pond saltern. Microb Ecol 11, 107-115.

Simon, R. D. (1981). Morphology and protein composition of gas vesicles from wild-type and gas vacuole defective strains of Halobacterium salinarium strain 5. J Gen Microbiol 125, 103-111.

Smith, D. F. (1928). The solubility of gases in solutions. In International Critical Tables of Numerical Data, Physics, Chemistry and Technology, pp. 271-283. Edited by E. W. Washburn. New York: McGraw-Hill.

Stoeckenius, W. \& Kunau, W. H. (1968). Further characterization of particulate fractions from lysed cell envelopes of Halobacterium halobium and isolation of gas vacuole membranes. J Cell Biol 38, 337-357.

Stoeckenius, W., Wolff, E. K. \& Hess, B. (1988). A rapid population method for action spectra applied to Halobacterium balobium. J Bacteriol 170, 2790-2795.

Surek, B., Pillay, B., Rdest, U., Beyreuther, K. \& Goebel, W. (1988). Evidence for two different gas vesicle proteins and genes in Halobacterium halobium. J Bacteriol 70, 1746-1751.

Walsby, A. E. (1971). The pressure relationships of gas vacuoles. Proc R Soc London, B 178, 301-326.

Walsby, A. E. (1973). A portable apparatus for measuring relative gas vacuolation, the strength of gas vacuoles, and turgor pressure in planktonic blue-green algae and bacteria. Limnol Oceanogr 18, 653-658.

Walsby, A. E. (1976). The buoyancy-providing role of gas vacuoles in an aerobic bacterium. Arch Microbiol 109, 135-142.

Walsby, A. E. (1994). Gas vesicles. Microbiol Rev 58, 94-144.

Walsby, A. E. \& Bleything, A. (1988). The dimensions of cyanobacterial gas vesicles in relation to their efficiency in providing buoyancy and withstanding pressure. J Gen Microbiol 134, 2635-2645.

Yang, C.-F. \& DasSarma, S. (1990). Transcriptional induction of purple membrane and gas vesicle synthesis in the archaebacterium Halobacterium halobium is blocked by a DNA gyrase inhibitor. J Bacteriol 172, 4118-4121.

Received 17 June 1996; revised 11 September 1996; accepted 13 September 1996. 